Journal of

Synchrotron

Radiation

ISSN 0909-0495

Received 10 December 2004

Accepted 22 March 2005
(C) 2005 International Union of Crystallography Printed in Great Britain - all rights reserved

\section{A high-throughput structural biology/proteomics beamline at the SRS on a new multipole wiggler}

\author{
Michele Cianci, ${ }^{\text {a,b }}$ Svetlana Antonyuk, ${ }^{a}$ Neil Bliss, ${ }^{a}$ Mike W. Bailey, ${ }^{a}$ \\ Stephen G. Buffey, ${ }^{a}$ Kan C. Cheung, ${ }^{a}$ Jim A. Clarke, ${ }^{a}$ Gareth E. Derbyshire, ${ }^{a}$ \\ Mark J. Ellis, a,b Mark J. Enderby, Alan F. Grant, ${ }^{a}$ Martin P. Holbourn, \\ David Laundy, ${ }^{a}$ Colin Nave, ${ }^{a}$ Roy Ryder, ${ }^{a}$ Paul Stephenson, ${ }^{a}$ John R. Helliwell ${ }^{\text {a,b,c }}$ \\ and S. Samar Hasnain ${ }^{a, b_{*}}$
}

\begin{abstract}
${ }^{\mathbf{a}}$ CCLRC Daresbury Laboratory, Warrington WA4 4AD, Cheshire, UK, ${ }^{\mathbf{b}} \mathrm{NWSGC}-$ North West Structural Genomics Center, Daresbury Laboratory, Warrington WA4 4AD, Cheshire, UK, and ${ }^{\mathbf{c}}$ Department of Chemistry, The University of Manchester, Oxford Road, Manchester M13 9PL, UK. E-mail: s.s.hasnain@dl.ac.uk
\end{abstract}

The North West Structural Genomics Centre's beamline, MAD10, at the SRS receives the central part of the radiation fan $(0.5 \mathrm{mrad}$ vertically, $4 \mathrm{mrad}$ horizontally) produced by a new $2.46 \mathrm{~T}$ ten-pole wiggler. The optical arrangement of the beamline consists of a Rh-coated collimating $\mathrm{Si}$ mirror, a fixed-exit-beam double-crystal monochromator with sagittal bending for horizontal focusing and a second Rh-coated Si mirror for vertical focusing. The double-crystal Si (111) monochromator allows data collection in the 5-13.5 keV photon energy range with rapid (subsecond) tunability and high energy resolution. The monochromatic beam is optimized through a $200 \mu \mathrm{m}$ collimator. The beamline end station has been designed around a Mar desktop beamline with high-throughput cryogenic sample changer, Mar225 CCD detector, liquid$\mathrm{N}_{2}$ autofill system and an ORTEC C-TRAIN-04 energy-resolving high-countrate $\mathrm{X}$-ray fluorescence detector. The instrument is optimized for MAD/SAD applications in protein crystallography with the additional mode of operation of online single-crystal EXAFS studies on the same crystals. Thus, screening of metals/Se in the crystal can be performed quickly prior to MAD/SAD data collection by exciting the crystal with X-rays of appropriate energy and recording an energy-dispersive fluorescence spectrum. In addition, this experimental set-up allows for parallel XAFS measurements on the same crystal to monitor 'radiation-induced' changes, if any, in e.g. the redox state of metal centres to be detected for a 'metallic' functional group during crystallographic data collection. Moreover, careful minimization of the thickness of the Be window maximizes the intensity performance for the 2.0-2.5 $\AA$ softer wavelength range. This range also covers the $K$-edges of a number of important $3 d$ transition metals as well as the $L$-edges of xenon and iodine and enhanced sulfur $f^{\prime \prime}$.

Keywords: structural genomics; high throughput; macromolecular crystallography; singlecrystal EXAFS; MAD; SAD; softer X-rays; metalloprotein; X-ray fluorescence.

\section{Introduction}

Over the last 30 years, X-ray crystallography and high-field NMR have transformed biology by providing three-dimensional structures of many important biological molecules and their complexes. Structure-function studies have provided unique insights into the working of complex biological systems. Protein function in the cellular context depends ultimately on the atomic structure and dynamics of individual proteins and their complexes. Enzyme specificity and mechanism, catalysis and regulation are just a few examples where atomic details of structures are required to provide insight into the detailed mechanisms by which cellular processes work and are regulated. During the last 20 years, synchrotron-based crystallography has expanded rapidly to fulfil this role and has become a major activity on every major synchrotron X-ray facility. The wavelength tunability allowed by synchrotron radiation sources has enabled the emergence of new phasing methods, namely multiple-wavelength anomalous dispersion (MAD) which involves three, four or five 
wavelengths (e.g. see Phillips \& Hodgson, 1980; Karle, 1980; Kahn et al., 1985; Hendrickson, 1991; Peterson et al., 1996; Hendrickson \& Ogata, 1997; Cassetta et al., 1999). Twowavelength methods (Okaya \& Pepinsky, 1956; Helliwell, $1979,1984)$ have recently grown in popularity, especially as data-set quality has steadily improved owing to better detectors and cryo-cooling of the sample (Peterson et al., 1996; González, 2003). Single, tuned-wavelength, anomalous diffraction (SAD) has also grown in popularity (Wang, 1985; Harvey et al., 1998; Yu-Dong et al., 1999; for a recent review, see Dauter et al., 2002; Dodson, 2003; Ramagopal et al., 2003; Olczak et al., 2003). These developments, together with the advent of selenomethionine substitution (Hendrickson et al., 1990), have transformed structure-determination endeavours bringing increasingly difficult problems to the experimental facilities and setting further challenges in the improvement of these experimental facilities. Between 1995 and 2003 the Protein Data Bank (PDB) deposition from the use of home sources (X-ray tubes etc.) have remained essentially constant while the PDB deposition from synchrotron radiation sources have increased by more than a factor of 25 . Thus, the total number of X-ray structures deposited in 2003 was close to 3000 whereas home sources contributed some 700 structures (Jiang \& Sweet, 2004).

Since the sequencing of human (The Genome International Sequencing Consortium, 2001), yeast (Wood et al., 2002) and mouse (Mouse Genome Sequencing Consortium, 2002) genomes, an ever-increasing number of genomes are being sequenced. The translation of genome sequences to large numbers of three-dimensional protein structures via a highthroughput structure-determination process has become of strategic importance for healthcare as well as fundamental biological science, requiring the development of highthroughput protein structure-determination facilities. Even though the average time lag between data collection at a synchrotron source and deposition of the structure in the PDB has gone down from 21 months in 1995 to 17 months in 2003 (Jiang \& Sweet, 2004), substantial effort is still required to improve this by a significant factor if we are to fully capitalize on the 'genomes sequencing' explosion.

It is estimated that at least about $30 \%$ of proteins coded by genomes are metalloproteins, i.e. utilizing the redox and ligand chemistry of metals to their advantage to perform varied biological functions with specificity and control. Quite often, only very small structural changes around the metal atom occur during complex biochemical reactions. A classic example is that of haemoglobin $(\mathrm{Hb})$, where a subtle change at the iron centre leads to major changes elsewhere in the protein structure and is responsible for the elegant allosteric mechanism of $\mathrm{Hb}$ (Perutz, 1970). The highest-resolution $\mathrm{Hb}$ $\mathrm{X}$-ray structure is now $1.5 \AA$ based on data collected at SRS station 9.6 (Waller \& Liddington, 1990). It is perhaps fair to claim that nowhere in the determination of molecular structure is precision more at a premium than in the case of metalloproteins. In this context the combined use of highresolution X-ray crystallography and X-ray absorption fine structure (XAFS) has proved extremely useful (Hasnain \&
Hodgson, 1999). The experimental synergy of MAD and XAFS has been pointed out previously (Hasnain \& Hodgson, 1999) but, despite this obvious compatibility, to our knowledge no experimental set-up exists where both MAD and highquality XAFS data can be collected on the same sample, until the development of the North West Structural Genomics Centre (NWSGC) beamline MAD10 at the SRS, reported here.

The realisation that the SRS could accommodate an additional insertion device has led to the high-field multipolewiggler-based MAD/XAFS capability at the SRS described here. It utilizes a rapidly tunable sagittally focusing monochromator, a high-count-rate energy-resolving fluorescence detector, an automatic cryogenic sample changer and a large $3 \times 3 \mathrm{CCD}$ detector. The full complement of protein crystallography facilities at the SRS have been recently reviewed (Cassetta et al., 1999; see also http://www.srs.ac.uk/srs/ stations.htm).

\section{Source characteristics and optical system}

\subsection{Choice of insertion device: multipole wiggler 10}

In order to provide a world-competitive MAD capability within the UK, the beamline has been built on a high-field $(2.46 \mathrm{~T})$ permanent-magnet multipole wiggler with an external gap of $20 \mathrm{~mm}$. The device is capable of providing a magnetic field of $3.2 \mathrm{~T}$ at $10 \mathrm{~mm}$ gap, a gap which is becoming the norm at third-generation sources. NWSGC MAD10 has been developed by a Daresbury inter-departmental project team. The 11-pole (nine full poles and two half poles) device, of period $220 \mathrm{~mm}$, is capable of providing an intense X-ray beam up to $14 \mathrm{keV}$, with a flux (at minimum gap) in excess of $10^{13}$ photons s $^{-1}(0.1 \% \text { bandwidth })^{-1}$ over the whole energy range of the instrument (see Fig. 1).

For this $1180 \mathrm{~mm}$-long device, three possible types of multipole wiggler (MPW) were considered. The energy of interest for this beamline was $12.7 \mathrm{keV}$, and maximizing the flux at this energy without compromising the 'vertical beam stay clear' (and so machine lifetime) was the main figure of merit. Achieving this energy required high on-axis peak fields from the insertion device. The three types of insertion devices

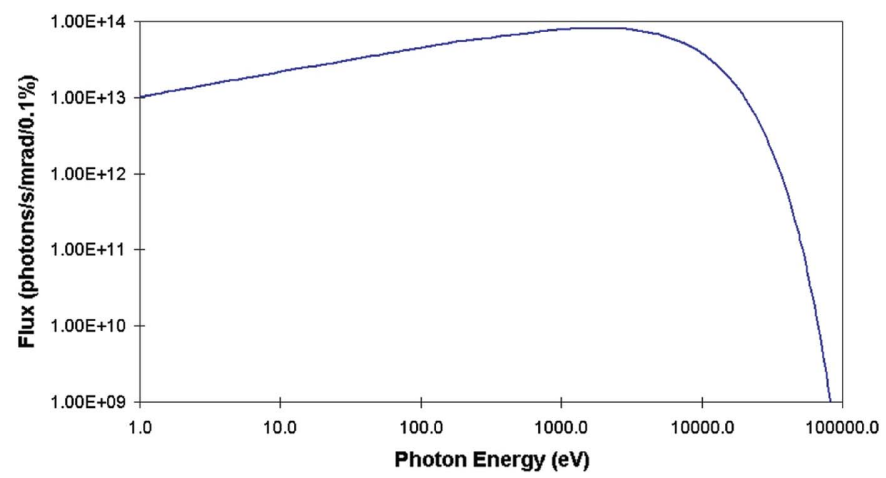

Figure 1

Integrated emitted flux over the central cone as a function of energy. 
Table 1

Comparison of two hybrid MPW designs currently working at the SRS including that on which the new beamline is based.

\begin{tabular}{lll}
\hline & $\begin{array}{l}\text { Existing MPW } \\
\text { at the SRS }\end{array}$ & $\begin{array}{l}\text { ESRF-based } \\
\text { MPW 10 }\end{array}$ \\
\hline Peak field (T) & 2.0 & 2.4 \\
Period (mm) & 200 & 220 \\
Gap (mm) & 20.5 & 20.0 \\
NdFeB remanent field, $B_{\mathrm{r}}(\mathrm{T})$ & 1.24 & 1.33 \\
Number of poles & 9 & 9 \\
Number of end poles & 2 & 2 \\
Flux at $12.7 \mathrm{keV} \mathrm{[photons} \mathrm{s}{ }^{-1}$ & $1.9 \times 10^{13}$ & $2.7 \times 10^{13}$ \\
$\quad$ mrad $^{-1}(200 \mathrm{~mA})^{-1}$ & & \\
$\left.(0.1 \% \text { bandwidth })^{-1}\right]$ & & \\
\hline
\end{tabular}

considered were a conventional hybrid design, an in-vacuum device and a superconducting MPW.

It was found that there was no significant advantage to be gained from an in-vacuum device over a conventional type. Significant work was required to understand how to deal with the transition between the vacuum vessel and the vertically adjustable magnet arrays. Designs in existence throughout the world have variable RF flaring that maintains a smooth conducting transition for the electron beam as the arrays are adjusted. This flaring can be quite long, especially in a case like the SRS where the stroke of the magnet needs to be large so that the magnet can be fully opened during injection at the lower beam energy. This solution was not practical given the short length of the straight section available and the budget and timescale constraints. A superconducting magnet design based on a recent example from MAX-II, assuming a current density of $500 \mathrm{~A} \mathrm{~mm}^{-2}$ in the superconducting coils, was explored. The preliminary design showed that the space constraints due to a neighbouring front end would severely limit the cryostat dimensions, making use of existing designs impractical. In addition, the cost and production time estimated for such a device precluded this option despite the advantage in flux gains. For these reasons the conventional hybrid MPW was chosen as the optimum choice.

The design for this device was scaled from the $3.1 \mathrm{~T}$ (at a gap of $11 \mathrm{~mm}$ ) ESRF asymmetric MPW (Chavanne et al., 1999). The device is of the asymmetric configuration; the period of $378 \mathrm{~mm}$ does not represent the period that should be assumed for a symmetric wiggler useful for the SRS. It was estimated, and subsequently proven, that if a period of $220 \mathrm{~mm}$ and a higher-remanent-field permanent-magnet material were used, a peak field of $2.4 \mathrm{~T}$ at a gap of $20 \mathrm{~mm}$ could be reached (as it turns out the device reached $2.46 \mathrm{~T}$ in practice), an improvement on the $2.3 \mathrm{~T}$ achieved by the ESRF device at the same gap. Table 1 compares the design based on this magnet with the two existing SRS MPW designs. To accommodate the minimum gap of $20 \mathrm{~mm}$ the straight section in the machine had to be modified with a narrow-gap vessel similar to that installed for the existing MPWs. The length of the device was maximized by removing some pumping from the straight section. In order to compensate for this, and owing to the narrower vessel, the inner walls of the insertion device were coated with NEG (non-evaporable getter) material, an alloy of titanium, zirconium and vanadium. This is thus the longest and the first NEG-coated device (achieved via collaboration with CERN) on the SRS. In order to achieve a very tight time scale, the MPW was designed, assembled and commissioned in-house.

\subsection{Optical layout of NWSGC MAD10}

The necessary requirements specified for the design of NWSGC MAD10 were for a high intensity on the sample whilst returning a low beam divergence at the sample $(<5 \mathrm{mrad})$, a high energy resolution $\left(10^{-4}\right)$, a beam size less than or equal to $200 \mu \mathrm{m}$, and rapid tunability over a wide photon energy range, $5-13.5 \mathrm{keV}$. In order to meet these requirements, four designs were studied by means of X-ray optics ray tracing.

All of the calculations were carried out for a Si (111) double-crystal monochromator with a Bragg angle of $11.406^{\circ}$ producing monochromatic radiation of energy $10 \mathrm{keV}$. The mirrors have a length of $1.2 \mathrm{~m}$ and the incidence angle on both mirrors was set to $3.5 \mathrm{mrad}$. The mirrors have a rhodium coating, and the resulting energy cut-off is above $15 \mathrm{keV}$. Calculations were performed for two configurations each using a mirror-double-bounce-monochromator-mirror arrangement.

One option was the combination of a vertically collimating mirror, double-bounce monochromator with sagittally bent second crystal for horizontal focusing, and vertically focusing mirror. Another option was a vertically collimating mirror and double-bounce (plane-crystal) monochromator with toroidal mirror for combined vertical- and horizontal-focusing arrangement.

The calculations were repeated for each configuration with the elements in the 2:1 (magnification 1/2) and 3:1 (magnification 1/3) horizontal-focusing settings, four options in all. A total of $20000 \mathrm{X}$-ray traces were used in each of the simulations. The source radiation was uniformly distributed over a vertical aperture of $0.40 \mathrm{mrad}$ and a horizontal aperture of $2 \mathrm{mrad}$ (no other assumption about the source of the radiation was made). An aperture in front of the first mirror was then used to reduce the fan to $0.32 \mathrm{mrad}$ vertically and horizontally to a width of $2 \mathrm{mrad}$ from the beamline centre line (Table 2).

From the calculations, higher flux is obtained with a sagittal monochromator in the 3:1 horizontal-focusing arrangement than with the corresponding arrangement using a toroidal mirror. In particular, for smaller sample size (50 $\mu \mathrm{m}$ aperture) the sagittal focusing provides almost a threefold increase over the toroidal mirror option. Thus, on the basis of maximizing the intensity at the sample, the 3:1 sagittal monochromator design option was chosen, in spite of the disadvantage of needing monochromator readjustments between absorption edges. The final beamline layout (Fig. 2) involves a vertically collimating mirror, double-bounce monochromator with sagittally bent second crystal for horizontal focusing, and a vertically focusing mirror. The focus predicted by ray tracing and that observed experimentally show close correspondence (Fig. 3). 


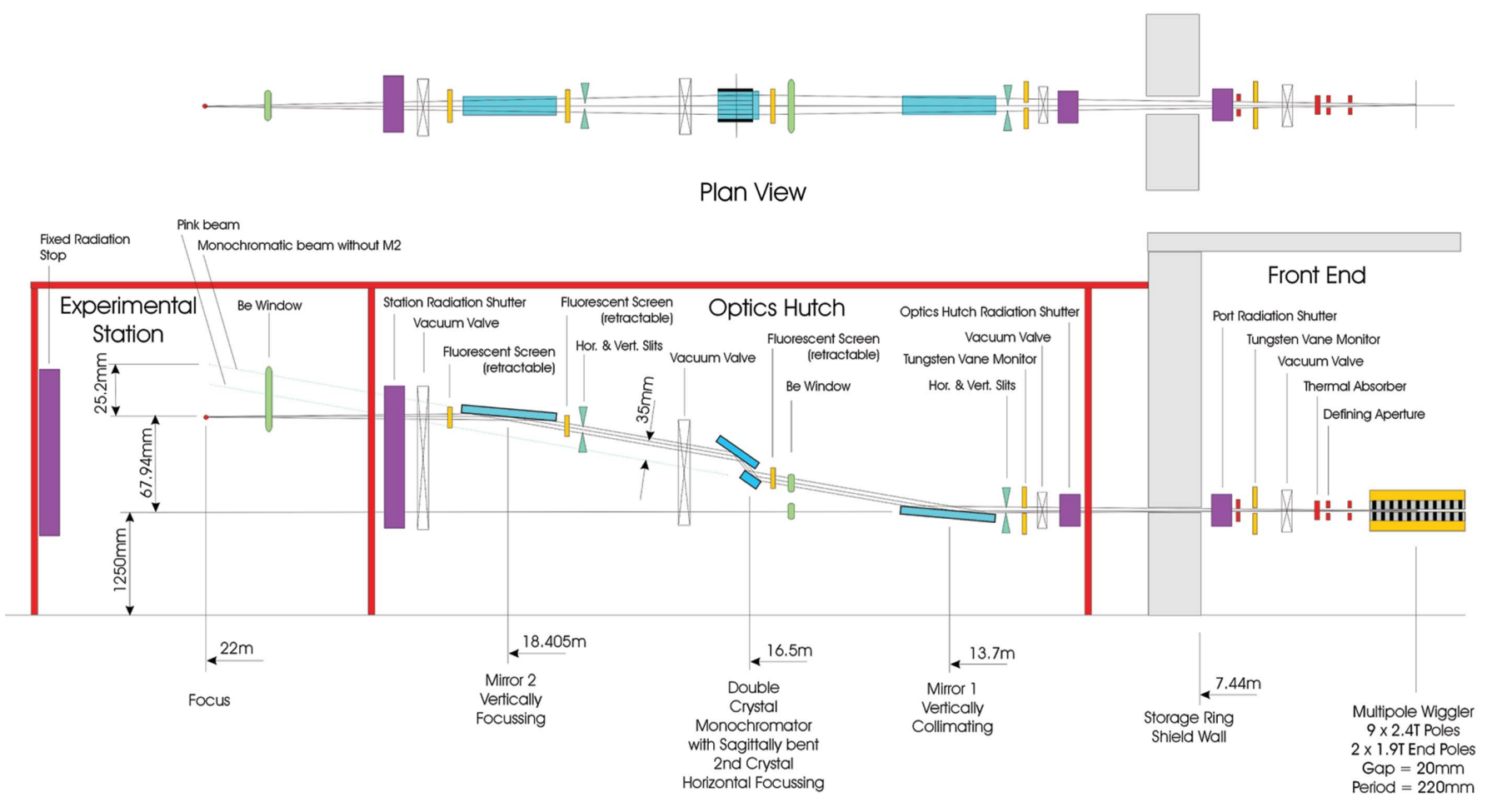

Figure 2

Schematic layout for the NWSGC MAD10 beamline at the SRS.

\section{Mechanical implementation}

\subsection{Front end}

Water-cooled apertures are positioned at various locations along the beamline front end to define the photon beam and protect the uncooled surfaces from the high heat load. The beamline horizontal- and vertical-defining aperture is positioned at $4.76 \mathrm{~m}$ from the MPW centreline source point and defines the horizontal distribution for the beamline at $3 \mathrm{mrad}$. The minimum vertical aperture was set at $\pm 0.75 \mathrm{mrad}$, which is available up to (and beyond) the exit flange of the inner-wall vacuum tube ( $9.429 \mathrm{~m}$ from the source point). The only other water-cooled horizontal and vertical aperture in the front is at $6.143 \mathrm{~m}$ from the source point. The front end is split into two vacuum regions. The first region extends from the bendingmagnet exit flange to the first all-metal valve at $4.9 \mathrm{~m}$ (part of the storage ring, sector 4, vacuum system). A vacuum of better than $1 \times 10^{-7} \mathrm{~Pa}$ with beam was obtained. The second region extends from the first all-metal valve to the exit flange of the end-wall vacuum tube. For this region, a vacuum of better than $1 \times 10^{-6} \mathrm{~Pa}$ with beam was obtained. In situ vacuum pumping was achieved by lumped pumping using ion pumps. A titanium sublimation pump was required in region 1 below the primary absorber vessel.

\subsection{Optics hutch}

3.2.1. Beam path. The synchrotron radiation beam, on entering the optics hutch, passes through a combined gas bremsstrahlung plus synchrotron radiation (GB/SR) shutter

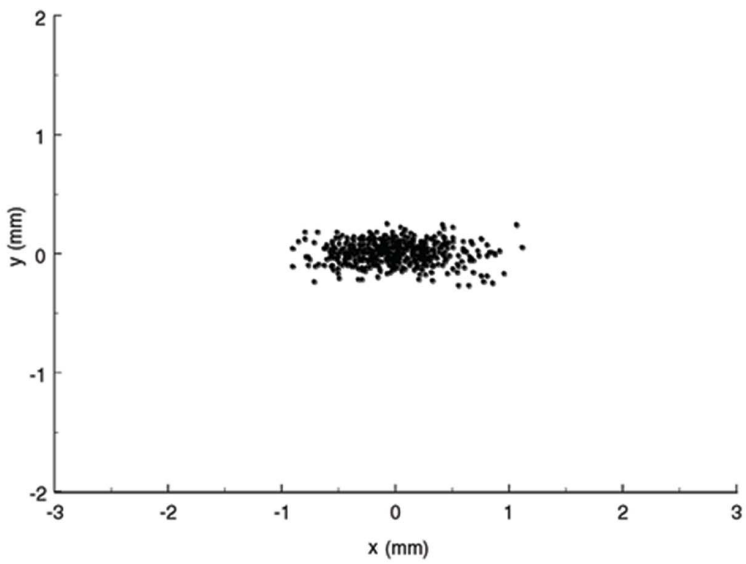

(a)

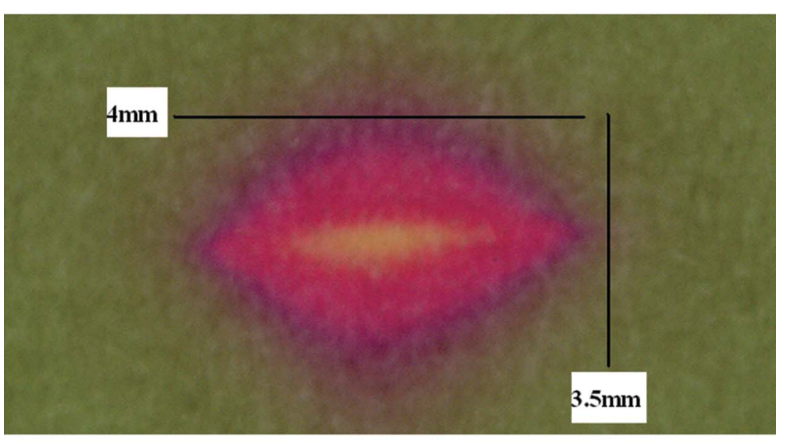

(b)

Figure 3

(a) Theoretical focused spot obtained for a 3:1 horizontal-focusing arrangement using a sagittally focusing crystal (see Table 2 for details), and $(b)$ experimental focused spot. In the experimental spot, the most intense portion is the orange-yellow $[1 \mathrm{~mm}(\mathrm{H}) \times 0.3 \mathrm{~mm}(\mathrm{~V})]$. 
Table 2

Parameters used and results of the ray tracing with 3:1 horizontal focusing for both the sagittal monochromator and the toroidal mirror options.

\begin{tabular}{|c|c|c|}
\hline & $\begin{array}{l}\text { Sagittal } \\
\text { monochromator } \\
\text { option }\end{array}$ & $\begin{array}{l}\text { Toroidal mirror } \\
\text { option }\end{array}$ \\
\hline Source-mirror 1 & $13.1 \mathrm{~m}$ & $13.1 \mathrm{~m}$ \\
\hline Mirror 1-monochromator & $3.55 \mathrm{~m}$ & $1.7 \mathrm{~m}$ \\
\hline Monochromator-mirror 2 & $1.93 \mathrm{~m}$ & $1.93 \mathrm{~m}$ \\
\hline Mirror 2-focus & $3.62 \mathrm{~m}$ & $5.577 \mathrm{~m}$ \\
\hline Source-focus & $22.2 \mathrm{~m}$ & $22.307 \mathrm{~m}$ \\
\hline Vertical magnification & 0.28 & 0.43 \\
\hline Horizontal magnification & $1 / 3$ & $1 / 3$ \\
\hline Mirror 1 bending radius & $7486 \mathrm{~m}$ & $7486 \mathrm{~m}$ \\
\hline $\begin{array}{l}\text { Monochromator bending } \\
\text { radius }\end{array}$ & $1.646 \mathrm{~m}$ & - \\
\hline Mirror 2 bending radius & $2069 \mathrm{~m}$ & $\begin{array}{c}3187 \mathrm{~m} \text { (longitudinal), } \\
29.0 \mathrm{~mm} \text { (sagittal) }\end{array}$ \\
\hline $\begin{array}{l}\text { Vertical focus spot size } \\
\text { from ray tracing (sigma) }\end{array}$ & $0.091 \mathrm{~mm}$ & $0.25 \mathrm{~mm}$ \\
\hline $\begin{array}{l}\text { Horizontal focus spot size } \\
\text { from ray tracing (sigma) }\end{array}$ & $0.36 \mathrm{~mm}$ & $0.28 \mathrm{~mm}$ \\
\hline $\begin{array}{l}\text { Flux into } 50 \mu \mathrm{m} \text { circular } \\
\text { aperture }\end{array}$ & 112 & 41 \\
\hline $\begin{array}{l}\text { Flux into } 100 \mu \mathrm{m} \text { circular } \\
\text { aperture }\end{array}$ & 444 & 179 \\
\hline $\begin{array}{l}\text { Flux into } 200 \mu \mathrm{m} \text { circular } \\
\text { aperture }\end{array}$ & 1410 & 682 \\
\hline $\begin{array}{l}\text { Total flux in focus } \\
\text { (arbitrary units) }\end{array}$ & 8729 & 7102 \\
\hline
\end{tabular}

mechanism and is required to access the optics hutch when the shutter is operated. The beam is steered using a tungsten vane monitor to ensure it is on the correct beam orbit plane. The beam is then defined vertically and horizontally at the entrance to the first mirror vessel using water-cooled vertical and horizontal beam-defining slit mechanisms. The beam is collimated using a water-cooled silicon mirror.

The beam then passes through a Be window at a source point distance of $15.6 \mathrm{~m}$ and into the sagittally focusing monochromator, which directs the beam into the second mirror vessel. The beam can be further defined vertically and horizontally at the entrance to the second mirror vessel using water-cooled vertical and horizontal beam-defining slit mechanisms. The beam access to the end station is controlled by the GB/SR shutter mechanism to allow users to enter the station when the shutter is closed. The beam finally passes through a Be window on the exit flange of a beam tube in the station at $21 \mathrm{~m}$ from the source point. The optics and endstation hutches are clad with $4 \mathrm{~mm}$-thick lead shielding sufficient to reduce external radiation from the hutches to a safe level. The optical-stage vacuum system has high vacuum with an average pressure of better than $1 \times 10^{-6} \mathrm{~Pa}$ with beam.

3.2.2. Mirror systems. The first mirror (M1) deflects the beam upwards at an angle of $3.5 \mathrm{mrad}$ and the second mirror (M2) deflects the beam at the same angle in the downwards direction, thus bringing the beam to the original, synchrotron radiation beam, direction. The mirror mount in each case accommodates a cooled silicon mirror which has dimensions of length $1200 \mathrm{~mm}$, width $120 \mathrm{~mm}$ and thickness $50 \mathrm{~mm}$. This M1 mirror receives a power loading of $6.5 \times 10^{-4} \mathrm{~W} \mathrm{~mm}^{-2}$ with the total power being $100 \mathrm{~W}$. Although this is only a small thermal loading, the M1 mirror is water-cooled to guarantee a time-dependent stability of the beam, a paramount design feature so as to work even with small anomalous dispersion signals. The cooling is achieved by placing two copper blocks in thermal contact with the side faces of the mirror. The copper blocks are connected by a copper pipe, through which water flows at a rate of up to $51 \mathrm{~min}^{-1}$. Both mirror systems have a number of motions so that their alignment can be controlled remotely.

3.2.3. X-ray double-crystal monochromator. To fully meet the design requirements of high flux, rapid (subsecond) tunability around an absorption edge, fine energy resolution and small spot size at sample, the design of choice for the monochromator was that developed for dilute-samples XAFS by Daresbury Laboratory, as described by Dobson (2002), already implemented on station 7.1 (Cheung et al., 1999) and more recently on station 6.2 (Cernik et al., 2004). This is, however, the first time that this design has been used in a beamline for protein crystallography.

The monochromator stage can be broken down into three main components: (i) the support block which supports the primary drive and vacuum vessel, (ii) the vacuum vessel, (iii) the primary drive itself.

The slides and vacuum vessel are mounted on a synthetic granite block which sits on three support jacks. These allow for the height and inclination of the primary drive to be set with respect to the synchrotron beam. The primary drive system is mounted in the welded fabrication of the vacuum vessel lid. The fabrication is a rigid structure which has been optimized for maximum stability. A highly accurate, reproducible and stable primary drive system is required for the double-crystal monochromator to accurately select the range of energy between 5 and $13.5 \mathrm{keV}$. The primary drive supports the $\mathrm{Si}$ (111) double-crystal system. It is capable of changing the Bragg angle of the first crystal with a resolution of less than 0.2 arcsec, yielding a $\delta E / E$ value of $2 \times 10^{-5}$ at $1.5 \AA$, better than the intrinsic energy resolution of $\mathrm{Si}(111), 1.33 \times 10^{-4}$, by more than an order of magnitude.

The first crystal of this double-crystal monochromator has a width of $90 \mathrm{~mm}$ and a length of $50 \mathrm{~mm}$. The diffracting surface is water-cooled via a single cylindrical bore. This ensures a constant temperature of the crystal surface and minimizes any decay of energy resolution/stability owing to beam-heating effects that would otherwise occur.

Optimum position of the second crystal is controlled by $X$ and $Z$ translations and by three drives for its roll, pitch and yaw. While changing the Bragg angle, conditions for reflection through the double-crystal system are the correct positioning of the second crystal within the 'sweet spot' of its curvature and its parallelism relative to the first crystal. These conditions are achieved by operating the pitch, yaw and roll motors. The $X$ and $Z$ translations ensure that the monochromatic beam emerging from the first crystal is reflected to the central part of the second crystal (which is where the curvature is most uniform) and exits the monochromator with constant vertical position as the Bragg angle is changed. The second $\mathrm{Si}$ crystal is 
Table 3

Overall X-ray absorption (\% of source intensity absorbed) for four options considered for the placements of Be windows and consequent total thickness of the windows.

Option 1 had two windows, one cooled window prior to the first mirror vessel and the second exit window prior to the desktop beamline. Option 2 had three windows, one on either side of the monochromator vessel and the final exit window. Option 3 had four windows isolating each of the optical elements and the final exit window. Option 4, the selected option, had two windows, the first between the first mirror vessel and monochromator and the final exit window.

\begin{tabular}{|c|c|c|c|c|c|c|c|}
\hline $\begin{array}{l}\text { Opt- } \\
\text { ion }\end{array}$ & $\begin{array}{l}\text { Total Be } \\
\text { thickness } \\
(\mathrm{mm})\end{array}$ & $\begin{array}{l}4 \mathrm{keV} \\
3.11 \mathrm{~A}\end{array}$ & $\begin{array}{l}6 \mathrm{keV} \\
2.07 \mathrm{~A}\end{array}$ & $\begin{array}{l}8 \mathrm{keV} \\
1.55 \mathrm{~A}\end{array}$ & $\begin{array}{l}10 \mathrm{keV} \\
1.24 \mathrm{~A}\end{array}$ & $\begin{array}{l}12 \mathrm{keV} \\
1.04 \mathrm{~A}\end{array}$ & $\begin{array}{l}14 \mathrm{keV} \\
0.89 \mathrm{~A}\end{array}$ \\
\hline 1 & 0.52 & 68 & 30 & 15 & 9 & 6 & 4 \\
\hline 2 & 0.45 & 62 & 26 & 13 & 8 & 5 & 3.6 \\
\hline 3 & 0.78 & 80 & 37 & 20 & 12 & 8 & 5.4 \\
\hline 4 & 0.35 & 52 & 22 & 11 & 6 & 4 & 2.8 \\
\hline
\end{tabular}

cut along the [111] direction and has a width of $90 \mathrm{~mm}$ and a length of $110 \mathrm{~mm}$. The horizontal focusing of the monochromatic beam is achieved by changing the bending of the second crystal with a curvature set according to the wavelength, i.e. a longer radius of curvature is required for lower-energy wavelengths. Ribs are included in the design of the crystal in order to reduce anticlastic bending of the crystal in the longitudinal direction. The crystal bender is designed to provide two opposing moments at the opposite edges of the crystal to ensure that the crystal bends into a cylindrical figure. The two $\mathrm{Si}$ (111) crystals were purchased from Crystal Scientific (UK) (www.crystal-scientific.com).

3.2.4. Be windows and beam visualization screens. The options (Table 3 ) for the Be-window positions were evaluated so as to achieve a minimum thickness of Be to allow maximum transmission of X-rays onto the sample, as well as to allow ease of commissioning the instrument and to safeguard the machine from the unlikely event of a window failure. The vertical opening of the window is a key parameter in reducing the stress and thus careful examination of this was undertaken. The option of a windowless beamline was rejected on the grounds of costs.

The option of choice consisted of a two-window arrangement, the first being $15.6 \mathrm{~m}$ downstream of M1. This assumed M1 to be effectively the first absorbing component in the beamline. The thermal absorption of the Be windows has been taken into consideration when analysing the thermal stress of the Be window. Window 2 was positioned at $21.9 \mathrm{~m}$ at the end of the beamline in the experimental station. This required a vacuum valve upstream of M1 and M2. In this case a total of $0.35 \mathrm{~mm}$ of $\mathrm{Be}$ intercepted the beam thus providing $80 \%$ transmission at $6 \mathrm{keV}(2.06 \AA)$.

In order to facilitate commissioning, pneumatically operated X-ray phosphor screens have been deployed in three positions. These are used as diagnostic devices and were particularly useful during the commissioning phase. They are positioned immediately upstream of the monochromator, and upstream and downstream of M2. They allow the user to visually check the beam (i) through the first Be window off M1, (ii) through the monochromator, (iii) as it reflects off M2.
The second screen is particularly useful during day-to-day optimization of the beam to visually check the correctness of the yaw and pitch positions of the second double-crystalmonochromator crystal.

\section{Experimental set-up}

\subsection{End station or experimental area}

The experimental area is characterized by three stand-alone pieces of equipment necessary to integrate the requirements for high-throughput structural genomics, screening for the presence of metals via XRF (thus contributing towards the identification of metalloprotein genes) and for combined online single-crystal XAFS/protein crystallography studies on the same crystal. They are described below.

\subsection{Marresearch desktop goniometer system}

The goniometer system is characterized by a high-precision $\varphi$-axis with exposure synchronization and shutter feedback. The collimator system (Fig. 4) is characterized by motorized slits, made of tungsten for high-quality beam-positioning shaping with variable aperture from 0 to $5 \mathrm{~mm}$ and $2.5 \mu \mathrm{m}$ positioning resolution. The rotary shutter is operated by a solenoid for fast operation $(1 \mathrm{~ms})$. The viewing direction of the mounted crystal is along the beam path $\left(20^{\circ}\right.$ offset) using a highly sensitive high-resolution CCD camera. The viewing area is $2 \mathrm{~mm} \times 2 \mathrm{~mm}$ and allows easy crystal location and centering (Fig. 4). This also allows crystal view image capture during data collection, potentially useful for analytical absorption correction especially given the interests in fostering softer X-ray anomalous dispersion applications (for a review see Djinović et al., 2005). Crystal mounting is also aided by a 4 " LCD monitor integrated into the goniometer. The tungsten beamstop is motorized with a $45 \mathrm{~mm}$ maximum crystal-to-beamstop distance and electronic detector collision protection.

An Oxford Instruments Cryojet mount is oriented at $45^{\circ}$ about the $\varphi$-axis (Fig. 4) with its gas stream centered within $0.5 \mathrm{~mm}$ around the crystal, the distance of the cryo-nozzle being adjustable from $4 \mathrm{~mm}$ to $18 \mathrm{~mm}$. The detector mount has a motorized crystal-to-detector distance translation stage with travel between 55 and $380 \mathrm{~mm}$. This is integrated into a motorized vertical $2 \theta$ arm $\left(0^{\circ}\right.$ to $30^{\circ}$ adjust) (Fig. 4). The combination of the $2 \theta$ arm with a Mar225 CCD detector and very short wavelengths $(\sim 0.9 \AA)$ will enable the station to collect data up to $0.65 \AA$ resolution at the corners (a $0.9 \AA$ resolution data set has already been collected on a metalloprotein). On the other hand, the combination of the $2 \theta$ arm with a Mar225 CCD detector and wavelengths up to $2.5 \AA$ will enable the station to collect data up to $1.8 \AA$ resolution at the corners.

\subsection{X-ray diffraction area detector}

A Mar165 CCD was installed for the initial commissioning phase. This was later replaced by a new $3 \times 3$ Mar CCD detector (Fig. 4) in the summer of 2004. This $3 \mathrm{k} \times 3 \mathrm{k}$ CCD is 


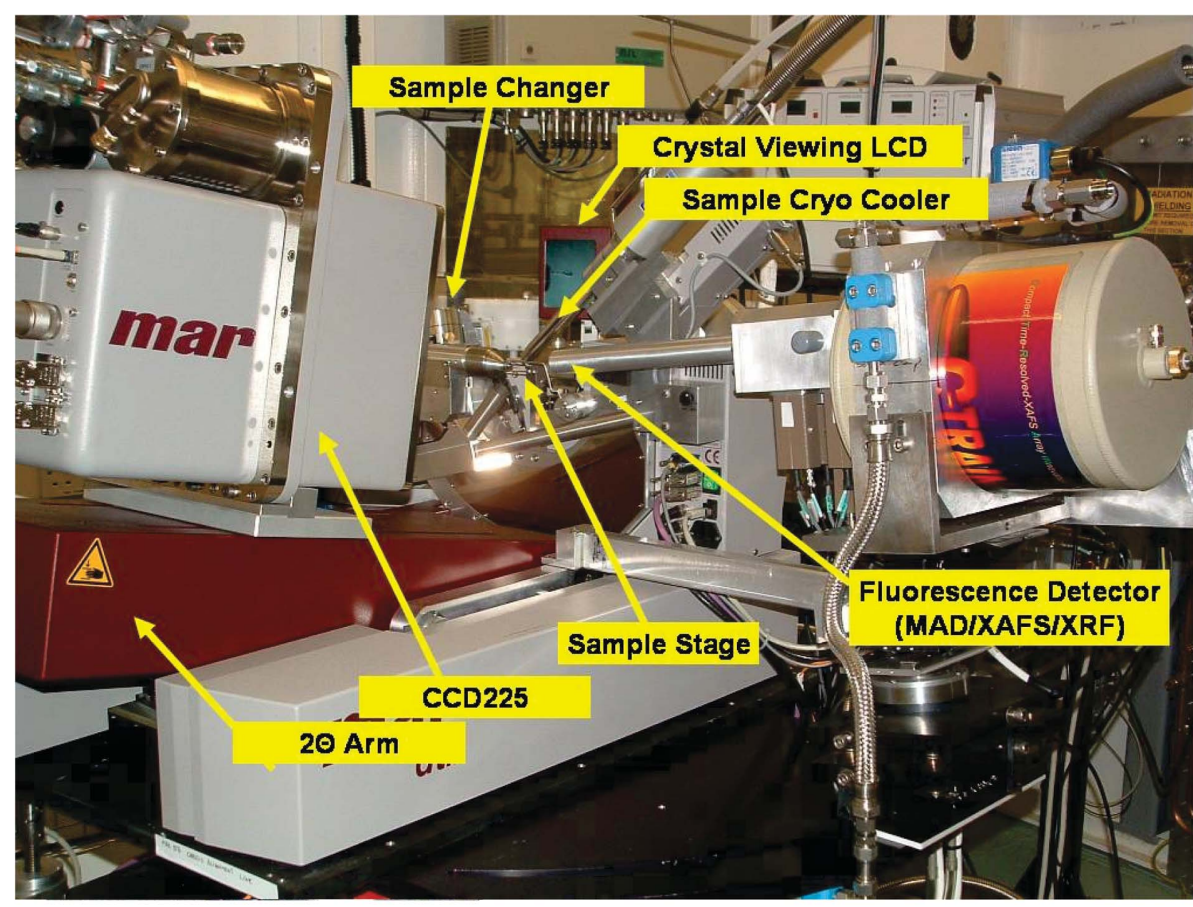

(a)

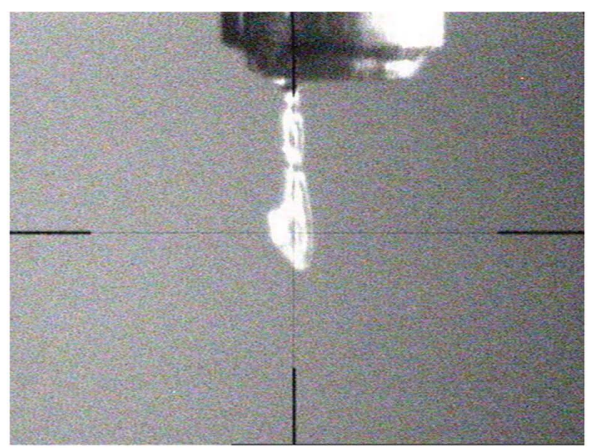

(b)

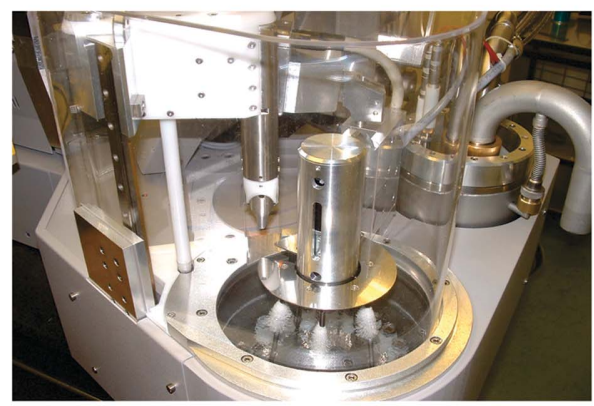

(c)

Figure 4

General view of the experimental end station. (a) Marreseach desktop beamline, Mar225CCD and ORTEC C-TRAIN-04 detector integrated. (b) Viewing a mounted crystal. The field of view is $2 \mathrm{~mm} \times 2 \mathrm{~mm}$. (c) Marreseach cryogenic sample changer.

optically coupled by relatively small tapers (taper ratio of 2.7:1) to provide a $225 \mathrm{~mm} \times 225 \mathrm{~mm}$ coverage. A relatively overall rapid read-out time of $4.5 \mathrm{~s}$, and a read-out noise of $8 \mathrm{e}$ pixel $^{-1}$ at $1 \mathrm{~s}$, allows higher-resolution data (weaker signal) to be collected. The $3 \times 3$ Mar CCD detector has two refrigerant-type coolers, switching relays and power supplies in a standard 19" rack. A floor-standing turbo pump maintains good vacuum in the detector head.

\subsection{Cryogenic sample changer and sample centering}

The Marreseach cryogenic sample changer (Fig. 4) is dedicated to automatic sample storage and exchange. The twochambered dewar allows for smooth refill of liquid nitrogen from a supplying dewar during operation. The carousel carries up to 19 samples and can easily be exchanged by hand in less than a minute. The system uses standard Hampton magnetic vials located in Teflon transfer containers that prolong the time before the liquid nitrogen starts boiling. The length and style of the pin holding the sample allows flexibility for future standards. The sample stays submerged in liquid nitrogen during the entire transfer from the carousel to the goniometer head, with the whole process lasting less than $2 \mathrm{~min}$.

Samples are centered using the integrated motorized $(x, y, z)$ goniometer head with a 'double-click' on the crystal as seen through the CCD camera. Each pin-holding cap can be tracked by a two-dimensional barcode applied to the surface of the caps. A barcode reader is integrated into the system. While in automatic mode only SPINE/Mar caps can be used; in manual mode the goniometer head can be quickly adapted to accommodate the flat Oxford Instruments caps and the standard Hampton caps.

\subsection{C-TRAIN-04 fluorescence detector}

In a collaborative project between CCLRC Daresbury Laboratory and ORTEC, a monolithic low-profile Ge detector has been manufactured (Fig. 4). Its general design is based on the proven C-TRAIN technology that is already in use at Daresbury Laboratory for XAFS and X-ray fluorescence (XRF) applications (Derbyshire et al., 1999). It provides the beamline with the capability of collecting fluorescence data for MAD experiments, and full range XAFS data on the same crystals. This provides the capability of monitoring the redox state of a 'metallic' functional group during crystallographic data collection. In addition, it allows for a trace-element analysis prior to beginning anomalous/heavy-atom derivative diffraction data collection. For a structural genomics programme, the XRF capability allows for routine screening of unknown proteins and thus can be used for the identification of metalloproteins.

The C-TRAIN-04 detector consists of a monolithic structure four-channel array with a $14.5 \mathrm{~mm}$ active diameter yielding an active area of greater than $95 \%\left(165 \mathrm{~mm}^{2}\right)$ of the total surface area. The monolithic array resides inside a cryostat snout of length $310 \mathrm{~mm}$ and diameter $30 \mathrm{~mm}$. The front active Ge surfaces are within $6 \mathrm{~mm}$ behind the front Be window. Additionally, miniaturization of the pre-amplifier resulted in a lower profile for the whole assembly than any of the large-solid-angle XRF solid-state detectors (Derbyshire 
Table 4

Statistics of three data sets collected on NWSGC MAD10.

The first two data sets have been obtained for two forms of crystals of the same protein, human superoxide dismutase. The third data set is an atomic-resolution data set of azurin II.

\begin{tabular}{llll}
\hline & HSOD & HSOD & Azurin II \\
\hline Space group & $C 222_{1}$ & $P 2_{1}$ & $P 4_{1} 22$ \\
$l(\AA)$ & 1.37 (first pass), & 1.07 & 0.98 \\
Exposure time (s) & $\quad 30$ (second pass) & 8 (quick pass) & 30 (slow pass), \\
& 0.5 (first pass), & 0.5 (slow pass), & 0.5 (slow pass), \\
Oscillation angle $\left({ }^{\circ}\right)$ & 1.0 (second pass) & 1.0 (quick pass) & 1.0 (quick pass) \\
Resolution range $(\AA)$ & $50-1.95$ & $50-1.24$ & 1.13 \\
Completeness $(\%)$ & $90.1(90.8)$ & $96.3(82.5)$ & $94.4(83.6)$ \\
$R_{\text {merge }}$ & $6.5(45.1)$ & $8.5(40.0)$ & $6.0(52.5)$ \\
$\langle I\rangle / \sigma I$ (last shell) & $52(2.3)$ & $20.5(2.0)$ & $19.8(2.1)$ \\
Redundancy & 8 & 4 & 8.0 \\
Overall reflections & 1341091 & 1116141 & 340835 \\
Unique reflections & 152998 & 70055 & 46365 \\
$a, b, c(\AA)$ & $166.18,203.59,145.58$ & $38.6,67.5,52.3$ & $51.8,51.8,99.8$ \\
$\alpha, \beta, \gamma\left({ }^{\circ}\right)$ & $90.0,90.0,90.0$ & $90.0,106.5,90.0$ & $90,90,90$ \\
\hline
\end{tabular}

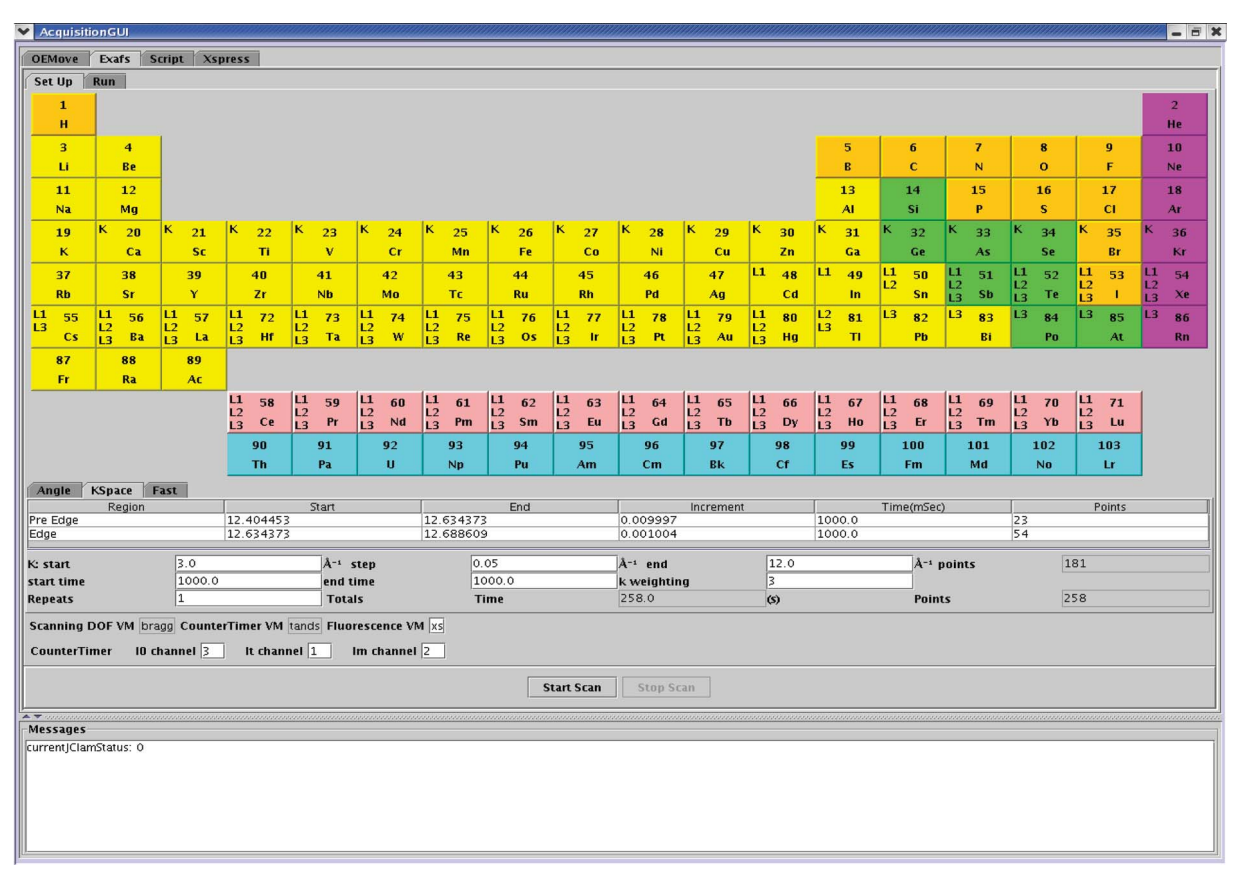

\section{Figure 5}

Snapshot of the software currently developed for beamline 10. The users decide the absorption edge of the element that they intend to investigate from the periodic table, and the graphical user interface will fill in the appropriate fields for the MAD/XAFS scan. high-quality XRF and consequently XAFS data. This detector, together with the XSPRESS (X-ray signal processing electronics for solid-state detectors) digital electronics developed at Daresbury Laboratory, provide a high-photon-count-rate capability in the region of several million photons per second.

\subsection{Beamline control software and electronics, data acquisition and analysis}

All the optical and beam-defining elements (slits, mirrors, monochromator and experimental table) are controlled by Parker/Digiplan controllers. The data-acquisition system for the C-TRAIN-04 fluorescence detector is VME-based with a Motorola processor running the OS9 operating system, a Daresbury Laboratory designed time frame generator, scaler and a voltage-tofrequency converter. These are used for general ion-chamber reading and transmission scan data. The graphical user interface (Fig. 5) communicates with the Parker control system and VME crate via an ethernet connection. A customized version of MarCCD software provided by Marresearch-MarUSA controls data acquisition with the Mar225 mosaic CCD detector, Mar desktop beamline camera and cryogenic sample changer.

\section{Benchmarking of instrument and first results}

\subsection{Native data sets}

First protein test images were et al., 1999) enabling integration with the Mar desktop beamline.

An energy resolution of $170 \mathrm{eV}$ has been achieved at a count rate per channel of well over $1 \times 10^{5}$ counts s $^{-1}$ using standard analog shaping amplifiers with a semi-Gaussian shaping time constant of $0.5 \mu$ s with X-rays of test energy $5.9 \mathrm{keV}$. The $250 \mu \mathrm{m}$-thick front Be window allows a usable energy range of above $3 \mathrm{keV}$ and, with a Ge crystal of active depth $6 \mathrm{~mm}$, this system has a good efficiency response and performance using X-rays of energy well above $50 \mathrm{keV}$.

The combination of compact detector snout, maximum ratio of active-to-total subtended solid angle, high count rate and high energy resolution is extremely valuable for obtaining obtained on a motor-neuron-causing mutant of human superoxide dismutase, as part of an on-going programme at Daresbury Laboratory (Strange et al., 2003; Hough et al., 2004; Antonyuk et al., 2005), with cell dimensions of $a=166.16, b=$ 203.68, $c=144.48 \AA$. Data extended to $1.8 \AA$ resolution and reasonable images were obtained in $45 \mathrm{~s}$ for $0.5^{\circ}$ oscillation. Table 4 provides the summary of two data sets obtained for two forms of crystals of the same protein, human superoxide dismutase. One of these is for an axis of $203 \AA$ and the other representing a relatively smaller cell (the largest axis being $67 \AA$ ). The atomic resolution achieved in the latter case and high resolution achieved in the former case provided early confirmation that the beamline had reached its basic specifi- 
Table 5

Data sets collected at NWSGC MAD10 during commissioning by users performing anomalous scattering experiments.

\begin{tabular}{|c|c|c|c|c|}
\hline & \multicolumn{4}{|l|}{ Type of experiment } \\
\hline & Se native & Se MAD & Mn anomalous & $\mathrm{S}$ anomalous \\
\hline Name of sample & N/A & $\mathrm{N} / \mathrm{A}$ & AMPcPP & ESCRT-II \\
\hline Space group & $P 622$ & $P 622$ & $I 222$ & $P 4_{3} 22$ \\
\hline$\lambda(\AA)$ & 0.979 & 0.979 & 1.8 & 2.070 \\
\hline Exposure time (s) & 15 & 15 & 30 & 15 \\
\hline Oscillation angle $\left({ }^{\circ}\right)$ & 1 & 1 & 1 & 1 \\
\hline Resolution range $(\AA)$ (last shell) & $33.4-2.9(2.95-2.8)$ & $39.5-3.2(3.37-3.2)$ & $30.0-2.4(2.5-2.4)$ & 4.2 \\
\hline Completeness (\%) (last shell) & $89.6(85.2)$ & $98.8(96.7)$ & $100(100)$ & $100(100)$ \\
\hline$R_{\text {merge }}$ (last shell) & $6.1(43.0)$ & $8.9(33.4)$ & $8.5(21.6)$ & $11.0(41.0)$ \\
\hline$\langle I\rangle / \sigma I$ (last shell) & $20.4(3.7)$ & $22.9(6.3)$ & $26.8(11.2)$ & $3.9(1.3)$ \\
\hline Redundancy & $6.7(5.6)$ & $10.2(8.1)$ & 10 & $24.9(24.0)$ \\
\hline Unique reflections & 16633 & 12498 & 42465 & 15969 \\
\hline$a, b, c(\AA)$ & $162.3,162.3,95.5$ & $162.3,162.3,95.5$ & $105.6,129.7,159.3$ & $150,150,186$ \\
\hline$\alpha, \beta, \gamma\left({ }^{\circ}\right)$ & $90,90,120$ & $90,90,120$ & $90,90,90$ & $90,90,90$ \\
\hline Reference & $\begin{array}{l}\text { F. Van den Ent et al. } \\
\text { (unpublished) }\end{array}$ & $\begin{array}{l}\text { F. Van den Ent et al. } \\
\text { (unpublished) }\end{array}$ & Mancini et al. (2004) & Teo et al. (2004) \\
\hline
\end{tabular}

cations and was able to provide high-quality crystallographic data, and that the beamline could enter into a detailed benchmarking stage while additional features of the line were commissioned.

Since March 2004 a number of groups have used the beamline as part of this benchmarking effort of the new beamline, and here some of those results are provided to indicate the performance of the instrument.

Azurin II has been implicated in the donation of electrons to nitrite reductases in denitrifying bacteria (Eady \& Hasnain, 2003). Azurin II exists as an eight-stranded $\beta$-barrel comprising two $\beta$-sheets that are sandwiched together. Several azurin II structures have already been published; however, K. Paraskevopoulos et al. (unpublished results) were able to collect the first atomic-resolution structure of this protein (Table 4, Fig. 6) which is being used for quantum mechanical calculations in order to establish features which determine their redox and electronic properties.

Tests on thrombin crystals showed a comparable resolution limit (1.3 ̊) for data from NWSGC MAD10 compared with an undulator-insertion-device-based instrument, although $I / \sigma(I)$ was higher for the undulator overall (Helliwell, Ahmed, Cianci \& Hubbard, unpublished).

\subsection{Data sets collected with anomalous signal}

One of the first experiments to be performed to identify a metal atom through the metal anamolous signal was performed by Mancini et al. (2004). They collected two data sets using $1.8 \AA$ X-rays, one with AMPcPP-Mn and one with AMPcPP-Mg, both at the same resolution of $2.4 \AA$ (Table 5). The anomalous difference map of the Mn data set revealed three $25 \sigma$ peaks (three molecules in the asymmetric unit) in close proximity to the AMPcPP molecules. The $2 F_{o}-F_{c}$ map of the $\mathrm{Mg}$ dataset revealed three peaks sitting exactly in the same positions as the Mn peaks. With that information they were able to demonstrate that these peaks were originating from bound $\mathrm{Mg}$ ions. We note that equivalent crystals diffract

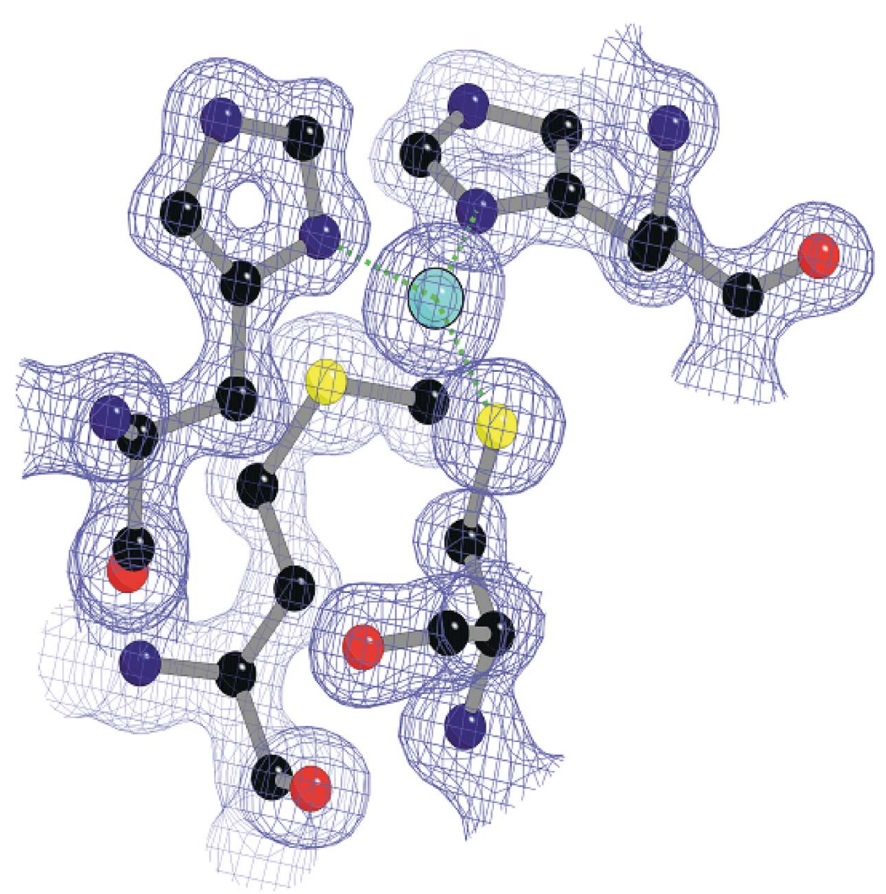

Figure 6

$2 F_{o}-F_{c}$ electron density map at $2 \sigma$ of azurin at $1.13 \AA$ resolution.

to similar resolution on BM14 of the ESRF (Mancini et al., 2004).

Dr J. Lowe and Dr F. Van den Ent from MRC-LMB, Cambridge, were the first to solve a structure using the MAD capability of the beamline. The C-TRAIN-04 fluorescence detector allowed them to perform quickly (less than $3 \mathrm{~min}$ ) a Se-edge scan and clearly identify the appropriate peak, edge and remote wavelength and collect high-quality data sets (Table 5) (F. Van den Ent et al., unpublished).

Dr R. W. Williams and co-workers from MRC-LMB recently reported the crystal structure of ESCRT-II, an endosome-associated complex required for protein sorting. For this, a high-multiplicity data set was collected using a 
wavelength of $2.07 \AA$. A $330^{\circ}$ data set was collected from a single crystal, with $15 \mathrm{~s}$ exposure per image with $1^{\circ}$ oscillation (Table 5). This yielded a 25 -fold multiplicity data set that gave a good sulfur anomalous differences electron density map up to a resolution of $4.2 \AA$, which provided landmarks to avoid making mistakes in interpreting a low-resolution structure. This data set allowed interpretation of the map for this $150 \mathrm{kDa}$ complex of three proteins (Teo et al., 2004).

\section{Directions and future developments}

\subsection{MAD and SAD phasing}

A case study on the application of softer X-rays to sulfur (Cianci et al., 2001) and xenon phasing (Olczak et al., 2003) has indeed driven the design specification of the softer X-ray aspect of this new MPW beamline. The new MPW beamline 10 , for high-throughput protein crystallography, is fitted with a Mar desktop beamline which allows a maximum $2 \theta$ of $110^{\circ}$ at maximum tilt and closest detector-to-crystal distance. Also, by careful minimization of the thickness of the beamline $\mathrm{Be}$ window, the intensity in the wavelength range $2-2.5 \AA$ is maximized. Thus, besides two-wavelength and MAD experiments from a wavelength of $0.9 \AA$ upwards, it is also feasible on SRS MPW MAD10 to collect high-throughput singlewavelength data sets, measured at a softer wavelength (SofterSWAT; Olczak et al., 2003). As a result, optimized xenon $L_{\mathrm{I}} f^{\prime \prime}$ and enhanced sulfur $f^{\prime \prime}$ signals, and signals from a number of other softer-wavelength edge anomalous scatterers (e.g. iodine), can also be obtained. This will extend the use of 'jolly SAD' applications (Dauter et al., 2002) to even more challenging cases, such as those involving fewer $\mathrm{S}$ atoms per $\mathrm{kDa}$ in the protein crystal asymmetric unit as well as weaker Xe substitution.

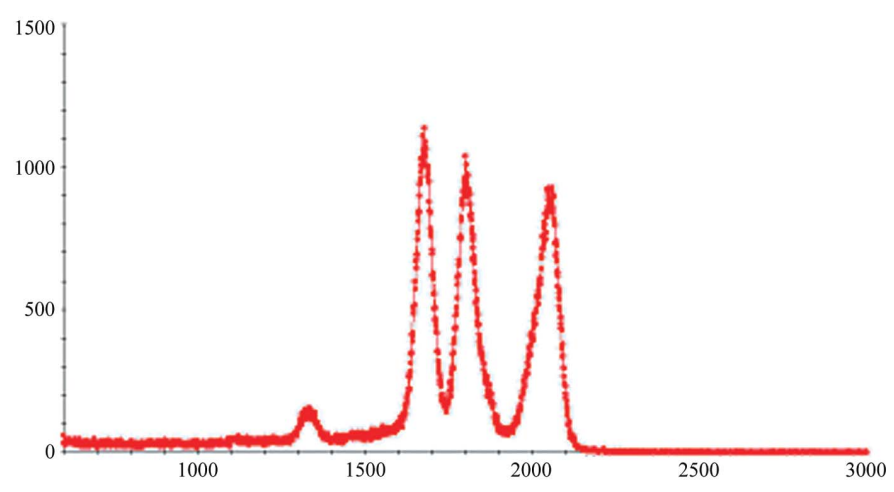

Figure 7

Application of the C-TRAIN-04 fluorescence detector: metal tracing on a protein derivative crystal with an exposure time of $15 \mathrm{~s}$. Ordinate: intensity of fluorescence signal (arbitrary scale); abscissa: energy (eV) of fluorescence (arbitrary scale). The first peak from the right is the scattered peak corresponding to the incident beam; the second and the third peak indicate the presence of $\mathrm{Zn}$ and $\mathrm{Cu}$, respectively.
6.2. Online metal analysis of protein crystals, metallogenomics and metalloproteomics

The ability to record high-quality energy-resolving fluorescence scans offers a number of new opportunities. Some of these are particularly important for high-throughput programmes of structural genomics and/or structural proteomics. Here we outline some possible examples.

For a hypothetical protein, XRF data collection using the energy-dispersive fluorescence detector on this MAD beamline would allow the experimenter to establish the presence of a metal atom in a matter of minutes without scanning over a large number of absorption edges (Fig. 7), which by necessity is time-consuming and exposes the crystal to an unnecessary radiation dose from which the crystal may not even survive for any diffraction data collection. The second scenario could be in establishing the presence of Se in the crystal without collecting diffraction data. This would allow much more efficient screening of crystals and is likely to prove very valuable in major structural genomics/proteomics programmes. The third example is screening of crystals, for example for heavy-atom derivative searches, where again the presence of a heavy atom can be identified from an XRF spectrum without scanning the monochromator or collecting diffraction data.

The above features are of particular importance to metalloproteins, which are expected to make up some $30 \%$ of a genome. Given that the identification of which genes code for metalloproteins by bioinformatics is a recognised challenge, routine screening of crystals of unknown proteins for metals is likely to prove helpful. In addition to the online screening for metals in hypothetical or known proteins, the ability to perform XAFS/XANES on the same crystal opens a new capability of knowing the redox state of the metal in a crystal for which diffraction data are also collected and its structure determined. In addition, the single-crystal XAFS capability allows one to exploit the polarization properties of the synchrotron radiation $\mathrm{X}$-rays and to collect angularresolved XAFS data capable of yielding three-dimensional information around the metal atom at subatomic resolution (Bianconi et al., 1985; Hasnain \& Hodgson, 1999). The combined use of X-ray crystallography and X-ray spectroscopy (XAFS) pioneered at Daresbury Laboratory (Hasnain, 2004; Hasnain \& Strange, 2003; Binsted \& Hasnain, 1996; Cheung et al., 2000) has already proved extremely powerful in a number of cases (Hasnain \& Hodgson, 1999). Such an approach for studying metalloproteins from a genome/ proteome would be facilitated by the availability of the new instrument.

\subsection{Other developments}

Crystal annealing has been used more and more in protein crystallography in recent years, sometimes with good success (see, for example, Ellis et al., 2002). Users have been annealing crystals by stopping the cooled nitrogen flow to the crystal basically by hand. In order to make this technique more 
reproducible, an online system for crystal annealing is being developed that allows the user to anneal a crystal remotely without having to enter the hutch in a time-controlled way (see §5.1).

Finally, the energy limits of the station can be extended at either end by altering the crystal gap or choosing a higherindex crystal. The current limitations are imposed by the monochromator crystal gap and the choice of $\mathrm{Si}$ (111), chosen to guarantee the highest flux possible and ease of operation with sagittal bend.

\section{Conclusion}

The initiative taken by the NWSGC scientists has allowed the development of a world-competitive combined high-intensity $\mathrm{MAD} / \mathrm{XAFS} / \mathrm{XRF}$ beamline in the UK for the wider structural biology community. The coupling of full XRF/XAFS capabilities with MAD offers a particular advantage of online screening of crystals for metals and heavy-atom derivatives and for combined single-crystal XAFS/protein-crystallography measurements.

The authors would like to thank M. Blum (Mar-USA) and A. Lentfer (Marresearch) for their collaboration in developing an integrated system and helping with the logistics of efficient commissioning of the beamline; Mr K. Paraskevopoulos (CCRLC, Daresbury) for supplying the data on atomic-resolution azurin II; Dr J. Lowe (MRC-LMB), Dr R. L. Williams (MRC-LMB) and Dr R. M. Esnouf (Oxford) for their useful feedback from benchmarking studies. We also thank the members of NWSGC and the beamline project team (see www.nwsgc.ac.uk for further details). We are also grateful to the members of the CCLRC's Biomedical College and the Molecular Biophysics Group for their interest and support. We also would like to thank Dr H. G. Price, Director of the CCLRC Daresbury Laboratory, at the start of the project, for his strong support. The members of the CCLRC Synchrotron Radiation Department executive board, the SRS operation team and engineering and technical staff are acknowledged for their constant support. We are indebted to Drs C. C. Tang, E. H. M. Duke and N. J. Terrill for sharing their experience of commissioning of beamlines. We are very grateful to BBSRC, MRC, EPSRC, NWDA and CCLRC for financial support (BBSRC grants 719/B15474 and 719/ REI20571 and NWDA project award N0002170).

\section{References}

Antonyuk, S., Elam, J. S., Hough, M. A., Strange, R. W., Doucette, P. A., Rodriguez, J. G., Hayward, L., Valentine, J. S., Hart, J. P. \& Hasnain, S. S. (2005). Protein Sci. In the press.

Bianconi, A., Congiu-Castellano, A., Durham, P. J., Hasnain, S. S. \& Phillips, S. (1985). Nature (London), 318, 685-687.

Binsted, N. \& Hasnain, S. S. (1996). J. Synchrotron Rad. 3, 185196.
Cassetta, A., Deacon, A. M., Ealick, S. E., Helliwell, J. R. \& Thompson, A. W. (1999). J. Synchrotron Rad. 6, 822-833.

Cernik, R. J., Barnes, P., Bushnell-Wye, G., Dent, A. J., Diakun, G. P., Flaherty, J. V., Greaves, G. N., Heeley, E. N., Helsby, W., Jacques, S. D. M., Kay, J., Rayment, T., Ryan, A., Tang, C. C. \& Terrill, N. J. (2004). J. Synchrotron Rad. 11, 163-170.

Chavanne, J., Van Vaerenbergh, P. \& Elleaume, P. (1999). Nucl. Instrum. Methods, A421, 352-360.

Cheung, K. C., Strange, R., Harvey, I., Dobson, B., Derbyshire, G., Kay, J., Binsted, N., Linford, R. \& Hasnain, S. (1999). J. Synchrotron Rad. 6, 161-163.

Cianci, M., Rizkallah, P. J., Olczak, A., Raftery, J., Chayen, N. E., Zagalsky, P. F. \& Helliwell, J. R. (2001). Acta Cryst. D57, 1219-1229.

Dauter, Z., Dauter, M. \& Dodson, E. (2002). Acta Cryst. D58, 494506.

Derbyshire, G., Cheung, K. C., Sangsingkeow, P. \& Hasnain, S. S. (1999). J. Synchrotron Rad. 6, 62-63.

Djinović Carugo, K., Helliwell, J. R., Stuhrmann, H. B. \& Weiss, M. S. (2005). J. Synchrotron Rad. 12, 000-000.

Dobson, B. R. (2002). Internal Technical Memorandum. Daresbury Laboratory, Warrington WA4 4AD, UK.

Dodson, E. (2003). Acta Cryst. D59, 1958-1965.

Eady, R. \& Hasnain, S. S. (2003). Denitrification in Comprehensive Coordination Chemistry II, Volume 8, Bio-Coordination Chemistry, edited by L. Que Jr and W. B. Tolman, pp. 759-786. Oxford: Elsevier Science.

Ellis, M. J., Antonyuk, S. \& Hasnain, S. S. (2002). Acta Cryst. D58, 456-458.

González, A. (2003). Acta Cryst. D59, 315-322.

Harvey, I., Hao, Q., Duke, E. H. M., Ingledew, W. J. \& Hasnain, S. S. (1998). Acta Cryst. D54, 629-635.

Hasnain, S. S. (2004). J. Synchrotron Rad. 11, 7-11.

Hasnain, S. S. \& Hodgson, K. O. (1999). J. Synchrotron Rad. 6, 852864.

Hasnain, S. S. \& Strange, R. W. (2003). J. Synchrotron Rad. 10, 915 .

Helliwell, J. R. (1979). Proceedings of the Daresbury Study Weekend, DL/Sci R13, pp. 1-6. Warrington: Daresbury Laboratory.

Helliwell, J. R. (1984). Rep. Prog. Phys. 47, 1403-1497.

Hendrickson, W. A. (1991). Science, 254, 51-58.

Hendrickson, W. A., Horton, J. R. \& LeMaster, D. M. (1990). EMBO J. 9, 1665-1672.

Hendrickson, W. A. \& Ogata, C. M. (1997). Methods Enzymol. 276, 494-523.

Hough, M. A., Grossmann, J. G., Antonyuk, S. V., Strange, R. W., Doucette, P. A., Rodriguez, J. A., Whitson, L. J., Hart, P. J., Hayward, L. J., Valentine, J. S. \& Hasnain, S. S. (2004). Proc. Natl. Acad. Sci. USA, 101, 5701-5702.

Jiang, J. \& Sweet, R. M. (2004). J. Synchrotron Rad. 11, 319-327.

Kahn, R., Fourme, R., Bosshard, R., Chiadmi, M., Risler, J. L., Dideberg, O. \& Wery, J. P. (1985). FEBS Lett. 179, 133-137.

Karle, J. (1980). Intl. J. Quantum Chem. 7, 356-367.

Mancini, E. J., Kainov, D. E, Grimes, J. M., Turna, R., Bamford, D. H. \& Stuart, D. L. (2004). Cell, 118, 743-755.

Mouse Genome Sequencing Consortium (2002). Nature (London), 420, 520 .

Okaya, R. \& Pepinsky, Y. (1956). Phys. Rev. 103, 1645-1647.

Olczak, A., Cianci, M., Hao, Q., Rizkallah, P. J., Raftery, J. \& Helliwell J. R. (2003). Acta Cryst. A59, 327-334.

Perutz, M. F. (1970). Nature (London), 228, 726-734.

Peterson, M. R., Harrop, S. J., McSweeney, S. M., Leonard, G. A., Thompson, A. W., Hunter, W. N. \& Helliwell, J. R. (1996). J. Synchrotron Rad. 3, 24-34.

Phillips, J. C. \& Hodgson, K. O. (1980). Acta Cryst. A36, 856-864.

Ramagopal, U. A., Dauter, M. \& Dauter, Z. (2003). Acta Cryst. D59, 1020-1027.

Strange, R. W., Eady, R. R., Lawson, D. \& Hasnain, S. S. (2003). J. Synchrotron Rad. 10, 71-75. 


\section{research papers}

Teo, H., Perisic, O., Gonzalez, B. \& William, R. L. (2004). Develop. Cell, 7, 559-569.

The Genome International Sequencing Consortium (2001). Nature (London), 409, 860-921.

Waller, D. A. \& Liddington, R. C. (1990). Acta Cryst. B46, 409418.

Wang, B. C. (1985). Methods Enzymol. 115, 90-112.
Wood, V., Gwilliam, R., Rajandream, M.-A., Lyne, M., Lyne, R., Stewart, A., Sgouros, J., Peat, N., Hayles, J., Baker, S., Basham, D., Bowman, S., Brooks, K., Brown, D., Brown, S. et al. (2002). Nature (London), 415, 871-880.

Yu-Dong, L., Harvey, I., Yuan-Xin, G., Chao-De, C. D., Yi-Zong, H., Hai-Fu, F., Hasnain, S. S. \& Hao Q. (1999). Acta Cryst. D55, 16201622. 